\title{
Positive effect of intravenous iron-oxide administration on left ventricular remodelling in patients with acute ST-elevation myocardial infarction - a cardiovascular magnetic resonance (CMR) study
}

\author{
Anca Florian ${ }^{1}$, Anna Ludwig ${ }^{2}$, Sabine Rösch², Handan Yildiz², Udo Sechtem², Ali Yilmaz ${ }^{1,2^{*}}$
}

From 17th Annual SCMR Scientific Sessions

New Orleans, LA, USA. 16-19 January 2014

\section{Background}

This study investigated the safety profile and potential "therapeutic" effect of intravenous ultrasmall superparamagnetic iron-oxide (USPIO)-based iron administration regarding infarct healing in patients with ST-elevation myocardial infarction (STEMI).

\section{Methods}

Seventeen study patients (IRON, $54 \pm 9$ yrs, $88 \%$ male) and 22 matched controls (CONTROL, $57 \pm 9$ yrs, 77\% male) both with primary reperfused STEMI underwent multi-parametric CMR studies in the first week and three months after acute MI. Only IRON patients received a single intravenous bolus of $510 \mathrm{mg}$ elemental iron as ferumoxytol (FerahemeTM) within four days following acute MI.

\section{Results}

Three months after study inclusion, all patients were alive and there were no adverse cardiac events. Significant improvement in left ventricular (LV) ejection fraction (IRON: $53 \pm 10 \%$ to $59 \pm 9 \%, \mathrm{p}=0.002$; CONTROL: $54 \pm 6 \%$ to $57 \pm 10 \%, \mathrm{p}=0.005$ ) as well as shrinkage of infarct size were seen in both groups at follow-up. There was a more pronounced decrease in infarct size in the IRON group (IRON: $-10.3 \pm 5.4 \%$ vs. CONTROL: $-7.0 \pm$ $8.4 \%, \mathrm{p}=0.050)$ in addition to a significant decrease in both endocardial extent and prevalence of transmural

\footnotetext{
${ }^{1}$ Cardiology and Angiology, University Hospital Münster, Münster, Germany
} Full list of author information is available at the end of the article infarctions in IRON but not in CONTROL patients. A significant decrease in LV end systolic volume was only seen in the IRON group $(71 \pm 25 \mathrm{~mL}$ to $59 \pm 25 \mathrm{~mL}$, $\mathrm{p}=0.002)$.

\section{Conclusions}

Intravenous iron administration in acute STEMI patients is associated with both improved infarct healing and beneficial global left ventricular remodelling (compared to matched controls). These findings together with the good safety profile make USPIO-based iron administration a promising future candidate as a "diagnostic" and "therapeutic" adjunctive solution in acute MI management.

\section{Funding}

This work was financially supported by a grant from the German Federal Ministry of Education and Research (BMBF; grant-ID 01EZ0818 to A.Y. and U.S.).

\section{Authors' details \\ ${ }^{1}$ Cardiology and Angiology, University Hospital Münster, Münster, Germany. ${ }^{2}$ Cardiology, Robert-Bosch-Krankenhaus, Stuttgart, Germany.}

Published: 16 January 2014

doi:10.1186/1532-429X-16-S1-O24

Cite this article as: Florian et al:: Positive effect of intravenous ironoxide administration on left ventricular remodelling in patients with acute ST-elevation myocardial infarction - a cardiovascular magnetic resonance (CMR) study. Journal of Cardiovascular Magnetic Resonance 2014 16(Suppl 1):O24. 